\title{
A Full-Duplex PON with Hybrid 64/16/4QAM OFDM Downlink and Hybrid 16/8/QPSK OFDM Uplink
}

\author{
Chao He, Zefu Tan, Yufeng Shao, Li Cai, Yunle Zhu, Hesheng Pu, Sisi Huang \\ College of Electronic \& Information Engineering, Chongqing Three Gorges University, Chongqing, China \\ Email: syufeng@163.com
}

Received 30 May 2016; accepted 21 August 2016; published 25 August 2016

\begin{abstract}
In the context of next-generation optical access networks beyond $10 \mathrm{G}$, for high SE and flexible dynamic bandwidth allocation (DBA), the scheme of hybrid 64/16/4QAM-OFDM signal for downlink transmission and hybrid 16/8/QPSK-OFDM signal for uplink transmission is successfully proposed and experimentally presented in a full-duplex PON based on OFDM system. Here, for the uplink, in order to unit management of the optical line terminal (OLT) and reduce cost, the optical source functioned as the optical subcarrier at optical network units (ONUs) is from OLT in the central station. Moreover, there is an external cavity laser (ECL) with center frequency of 193.2 THz not only employed as optical modulated signal but also acted as LO signal. Our simulation results show that bit error ratio (BER) under hardware detection forward error correction has been successfully gained after $20 \mathrm{~km}$ of SSMF transmission. It is observed that the receiver sensitivity of multilevel PSK (M-PSK) is obviously larger than that of the M-QAM in this measurement scheme.
\end{abstract}

\section{Keywords}

Passive Optical Network, Digital Signal Processing, Full-Duplex, Hybrid Modulation, Orthogonal Frequency Division Multiplexing

\section{Introduction}

Over the past decades, for both developing wider, faster, and smarter access network and providing subscribers with best services experience, such as internet protocol television (IPTV), high definition television (HDTV), video-on-demand (VOD), virtual private network (VPN), cable television (CATV), and surfing the Internet, passive optical network (PON) has been successfully proposed and experimentally demonstrated for the next generation broadband optical access system [1]. To meet the ever-increasing bandwidth requirements of the further future optical access systems, a large number of PON architectures have been successively introduced and presented. Firstly, time division multiplexing PON (TDM-PON) such as broadband PON (BPON), Ethernet PON (EPON) and gigabit PON (GPON), which is the most significant energy efficiency optical access method currently deployed in fiber-to-the-x (HTTx), connects the optical line terminal (OLT) with the multi-optical network units (ONUs) with the help of the optical distribution network(ODN) in the form of point-to-multipoint [2]. 
Furthermore, wavelength division multiplexing PON (WDM-PON) is another scheme having been widely investigated because of high bandwidth and low latency, where the passive splitter has been replaced by arrayed waveguide gratings (AWG) [3]. Consequently, hybrid PON (HPON) such as time and wavelength PON (TWDM-PON), which can be emerged as a primary solution for the next generation passive optical stage 2(NG-PON2) by the full service access network (FSAN) community in the April 2012, stacking multiple pairs of wavelengths into XG-PONs [4]. Then, frequency division multiplexing PON (FDM-PON) is pretty different from OFDM-PON, which has also been explored for access networks, with low computational complexity and peak-to-average power (PAPR) reduction [5] [6]. Specifically, for the sake of transmission capacity enhancement of high speed optical systems and networks, the transmission of mode division multiplexing-based PON (MDM-PON) system over few-mode fiber (FMF) is discussed, in which modal crosstalk is one of the most critical issues [7].

Recently, orthogonal frequency division multiplexing (OFDM) scheme regarded as a multicarrier modulation (MCM) technique has been widely considered as a very promising candidate for either wireless communication in 1909 or optical fiber communication (OFC) in 2009 owing in large part to high spectral efficiency, flexible dynamic bandwidth allocation, outstanding tolerance against chromatic dispersion (CD) and polarization mode dispersion (PMD), evident improvement of the transmission rate, simple channel equalization, and high-level modulation format [8]-[13]. In order to counteract the dispersion of OFC posed by larger transmission capacity, PON combined with OFDM scheme has been widely used in broadband access "last mile" [9]. For physical layer security enhancement and PAPR reduction, varies techniques based on chaotic scheme have been described in OFDM-PON system, including chaos IQ-encryption-based optimal frame transmission (IQ-OFT) technique [10], and analog-digital hybrid chaotic system [11]. An OLT receiver based on both an optical coherent detection and a fixed-gain front-end electrical amplifier in OFDM/TDMA-PON system was investigated, which was able to normalize the power of the upstream burst OFDM signal [12]. R. B. Nunes et al. demonstrated the SE of the bandwidth scalable OFDM PON (BSOFDM-PON) system with subcarrier emphasis was enabled by advanced modulation format and simple channel compensation [13].

In this paper, we successfully analyzed a full-duplex PON access technique for both high SE and flexible dynamic bandwidth allocation in in-phase/quadrature-phase modulation coherent detection OFDM (IQ-CO-OFDM) system, which consisted of two 40-Gb/s links signal transmitted over 20-km optical fiber path of SMF-28. One is hybrid 64/16/4QAM OFDM signal used for the downlink transmission and the other is hybrid 16/8/QPSK OFDM signal available for the uplink transmission. For the sake of cost effective and complexity reduction, an ECL at $193.2 \mathrm{THz}$ has been successfully found for both downlink to provide an optical carrier signal with the ONU and uplink to perfectly generate LO signal. It is necessary for us to study how to realize subcarrier allocation in IFFT/FFT based on passive optical access system.

\section{Principle of Hybrid Modulation}

As we know, PON is of great importance in optical access network, which concluding none electrical component and electronic power supply in optical distribution network (ODN). As Figure 1 shows that point to multi points (P2MP) has been a promising candidate for the PON among a large number of schemes, in which, the front end devices functioned OLT communicate with a few customer premise equipment (CPE) proposed of ONUs/ONTs by passive optical cable with SSMF and passive power splitter/coupler (PPSC).The situation of free-source in the ODN can avoid electromagnetic interference stemming from external devices, decrease failure rate between line and device, promote system reliability , and cut down maintenance cost in the next generation passive optical network.

The basic OFDM modulation and demodulation principle based on hybrid 64/16/4-QAM signal and hybrid 16/8/Q-PSK signal can be depicted in Figure 2 in detail, in which, the inset stands for the block design of subcarrier allocation for hybrid modulation technology. More specifically, among all the 128 subcarriers, namely, the number of IFFT/FFT are 128, 120 subcarriers are efficiently transmitted useful data, others 8 subcarriers are automatically set to zero. For reference, subcarriers arranging from 5 to 44, from 45 to 84, and from 85 to 124 are simultaneously allocated to 4QAM, 16QAM, and 64QAM, respectively, while, the same location subcarriers are assigned to QPSK, 8PSK, and 16PSK, respectively. However, channel estimation and synchronization technique has been widely applied in OFDM receiver end to efficiently resist multipath fading imposed by inter-carrier interference (ICI) and inter-symbol interference (ISI), where the situation is the same as that the optical signal based on OFDM (OOFDM) can contradict dispersion in the fiber channel. 


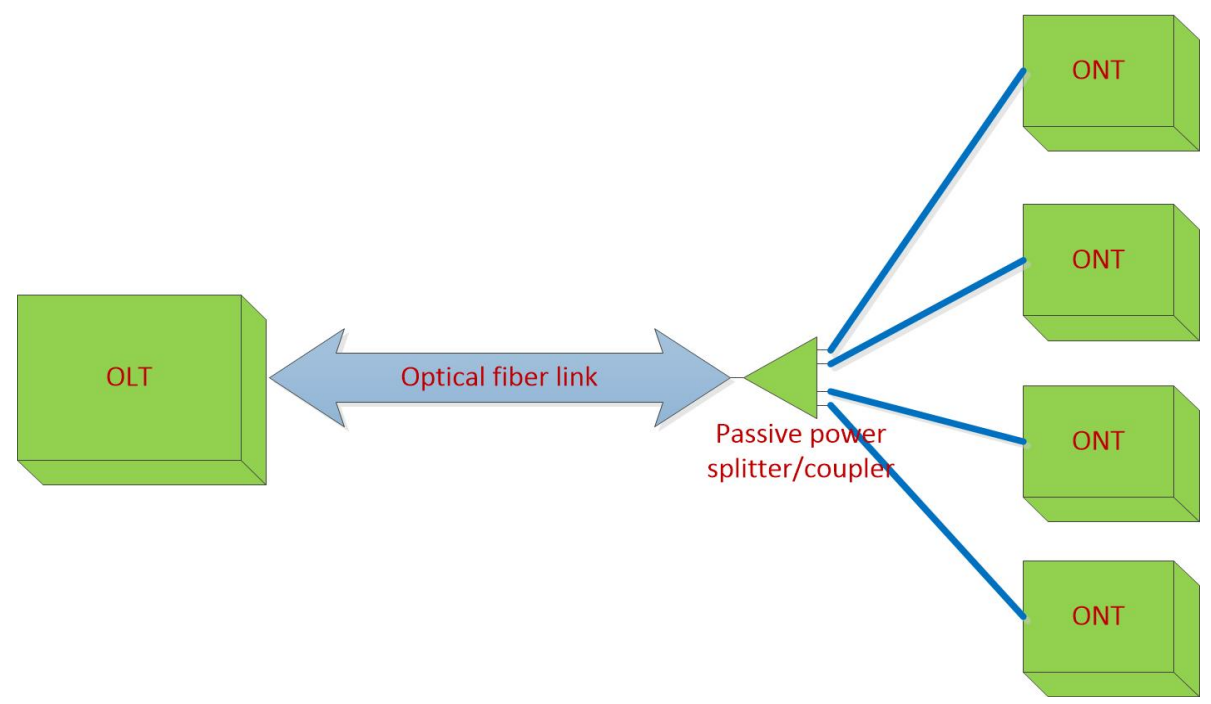

Figure 1. Block diagram of broadband optical access system.
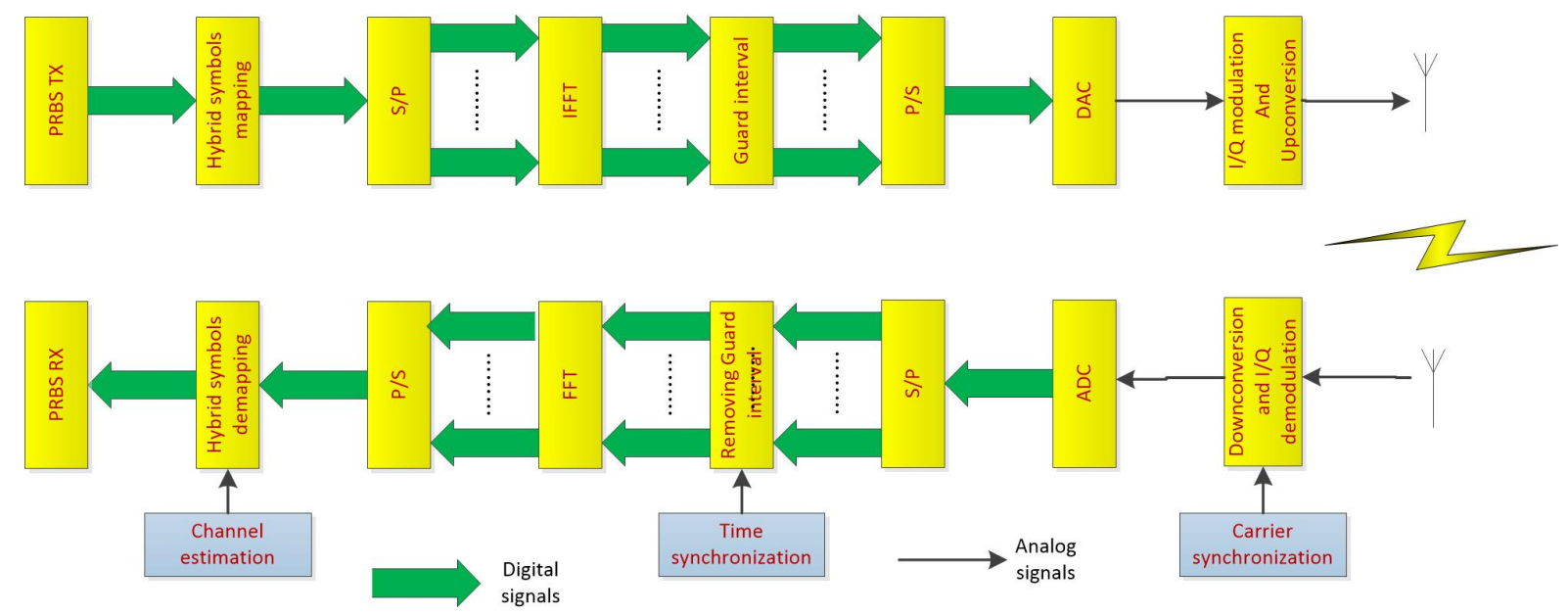

Figure 2. Block diagram of OFDM transceiver.

\section{System Simulation for $40 \mathrm{~Gb} / \mathrm{s}$ Hybrid OFDM-PON}

The simulation setup of the full-duplex OFDM-PON for both hybrid 64/16/4QAM OFDM downlink signal and hybrid 16/8/QPSK signal transmission is shown in Figure 3. For downlink transmission part, at the central station (CS), the continuous wavelength (CW) light-wave functioning as the optical carrier signal with wavelength of 193.1-THz from ECL1 possessing a power of $10-\mathrm{dBm}$, a linewidth of $0.1 \mathrm{MHz}$ and an initial phase of 0 degree can be first modulated by 40-Gb/s hybrid 64/16/4QAM electrical radio frequency (RF) OFDM signal plotted in the inset of Figure 3 into the corresponding modulated optical baseband OFDM signal in the RF to optical up-converter (RTO), which a polarization controller to split optical carrier signal into two branches, two parallel LiNb Mach-Zehnder Modulators (MZM) operated at null bias point provided with an extinction ratio (ER) of $60-\mathrm{dB}$, a bias voltage of $4 \mathrm{v}$, and a insertion loss of $1 \mathrm{~dB}$ to transform the in-phase and quadrature-phase electrical OFDM signal into in-phase and quadrature optical OFDM signal with the help of the upper branch and lower branch optical carrier, a phase shifter with 90 degree phase shift to add a time phase advance/delay to the input optical signal, and an optical coupler to recombine the two branches OOFDM signal into one branch, then coupled by a coupler to integrate the hybrid 64/16/4-QAM modulated OOFDM baseband signal and the optical signal with 193.2-THz wavelength from ECL2 into the mixing optical signal , and finally magnified by an EDFA operating at gain control mode owning gain and noise figure (NF) are 10- and 4-dB,respectively. The generated hybrid frequency signal is successfully launched into 20-km SSMF, which has attenuation factor of 


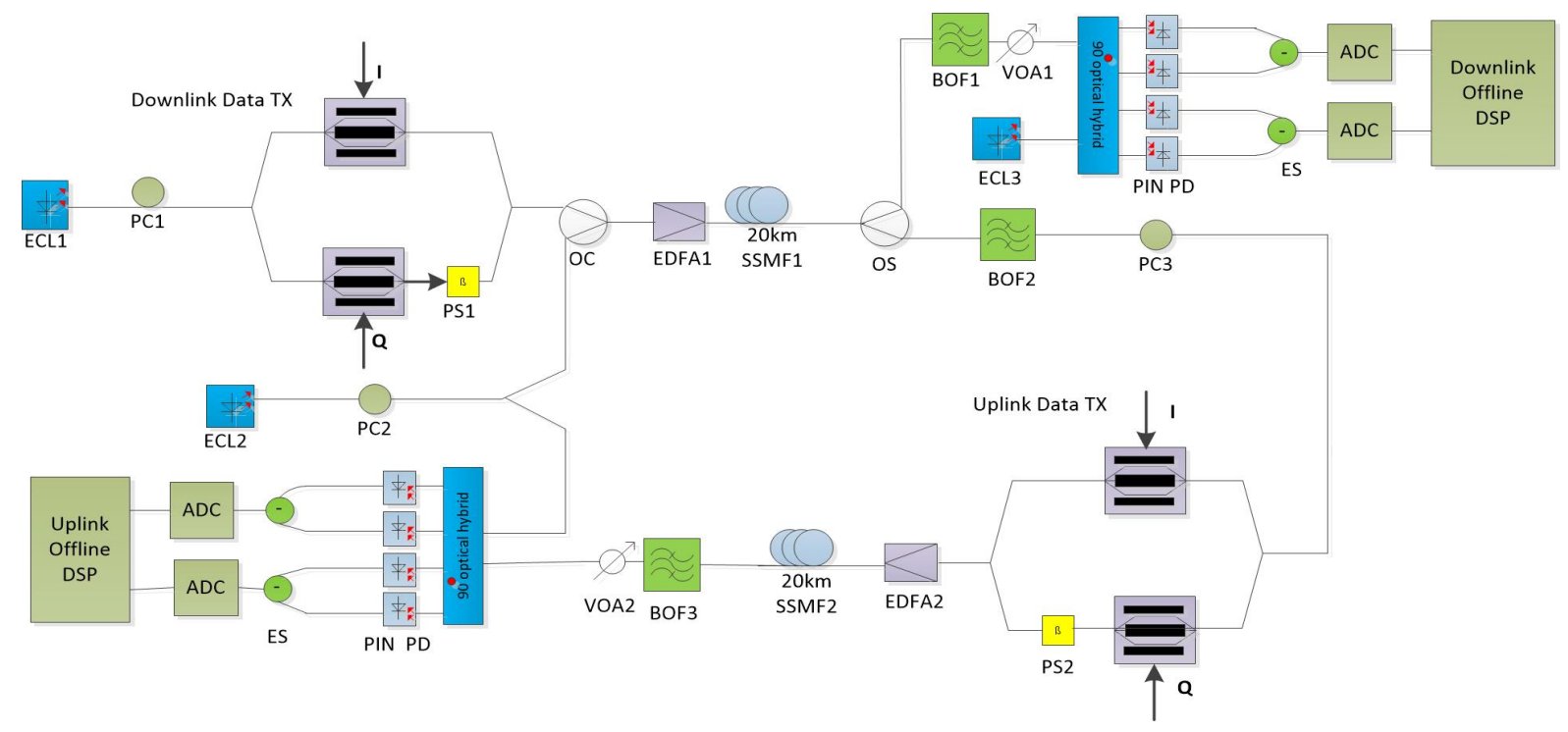

Figure 3. Experimental setup for the full-duplex OFDM-PON.

0.2-dB/km, chromatic dispersion (CD) coefficient of $16.7-\mathrm{ps} / \mathrm{nm} / \mathrm{km}$ and polarization mode dispersion (PMD) coefficient of $0.05-\mathrm{ps} / \mathrm{sqrt}(\mathrm{km})$ and constant effective area data type. Subsequently, a passive optical splitter (POS) is adopted to divide the received optical signal into two branches signals.

At the base station (BS), one branch signal is first launched into Bessel optical filter with (BOF) with center frequency of 193.1-THz, 3-dB bandwidth of $40 \mathrm{GHz}$, and filter order of 3.Then, in order to change received optical power of the ONUs, it is highly desirable that the variable optical attenuator (VOA) between BOF1 and coherent detection is as simple as possible. Afterwards, the received signal is coherent detected with the local oscillator (LO) signal from ECL3 with the center frequency the same as that of the ECL1 in the coherent detector in the form of homodyne detection, which a 90 degree optical hybrid to use coherent signal demodulation and make two input signal into four output signal , four parallel PIN Photodiodes with responsivity of 1-A/W, modulation bandwidth 2-GHz, and sample rate of $40-\mathrm{GHz}$ to transform the optical signal into electrical signal , two parallel electrical sub-tractor to subtract the four electrical signal into in-phase signal and quadrature signal, two parallel electrical amplifier (EA) with additive thermal noise (ATN) to promote the amplitude of the electrical signal. Finally, it should be mentioned that offline DSP in the OFDM demodulation, which concluding analog to digital conversion (ADC) accomplished by down-sampling to the global symbol rate, dispersion compensation implemented by digital filter, removing cyclical prefix (CP) to reduce the ISI and ICI, FFT to transform the discrete time domain signal into the discrete frequency domain signal, training symbols-based channel estimation, pilot symbols-based carrier phase estimation.

For uplink part, optical carrier signal after BOF2 with center frequency different from that after BOF1 is first modulated with the generated RF hybrid 16/8/QPSK OFDM electrical signal at the BS, which has the same functions as the downlink I/Q modulation. In order to match the launched optical power (LOP) transmitted over the exactly same SSMF used for downlink, the perfect combination of both EDFA2 and VOA2 can be used to bridge the power penalty between the transmitter and the receiver to achieve high system performance. On the other hand, at the receiver side, functioned as optical carrier signal from ECL1 has been coherently detected with the hybrid modulation optical baseband signal. From the perspective of the SSMF deployed from the BS to the CS, the situation of B2B is independent of 20-km fiber transmission. Finally, offline DSP has been experimentally investigated to implement OFDM demodulation.

\section{Simulation Results and Discussion}

As Figure 4 demonstrates the optical spectrum of different location in this scheme, the optical spectrum before OC is shown in Figure 4(a), which explains the transmission signal with power of 25-dBm and bandwidth of 10 GHz. Figure 4(b) illustrates the optical spectrum after OC, where both 100-GHz frequency spacing and 30-dBm power difference exist in modulated optical baseband signal at 193.1-THz and LO signal at 193.2-THz. The 

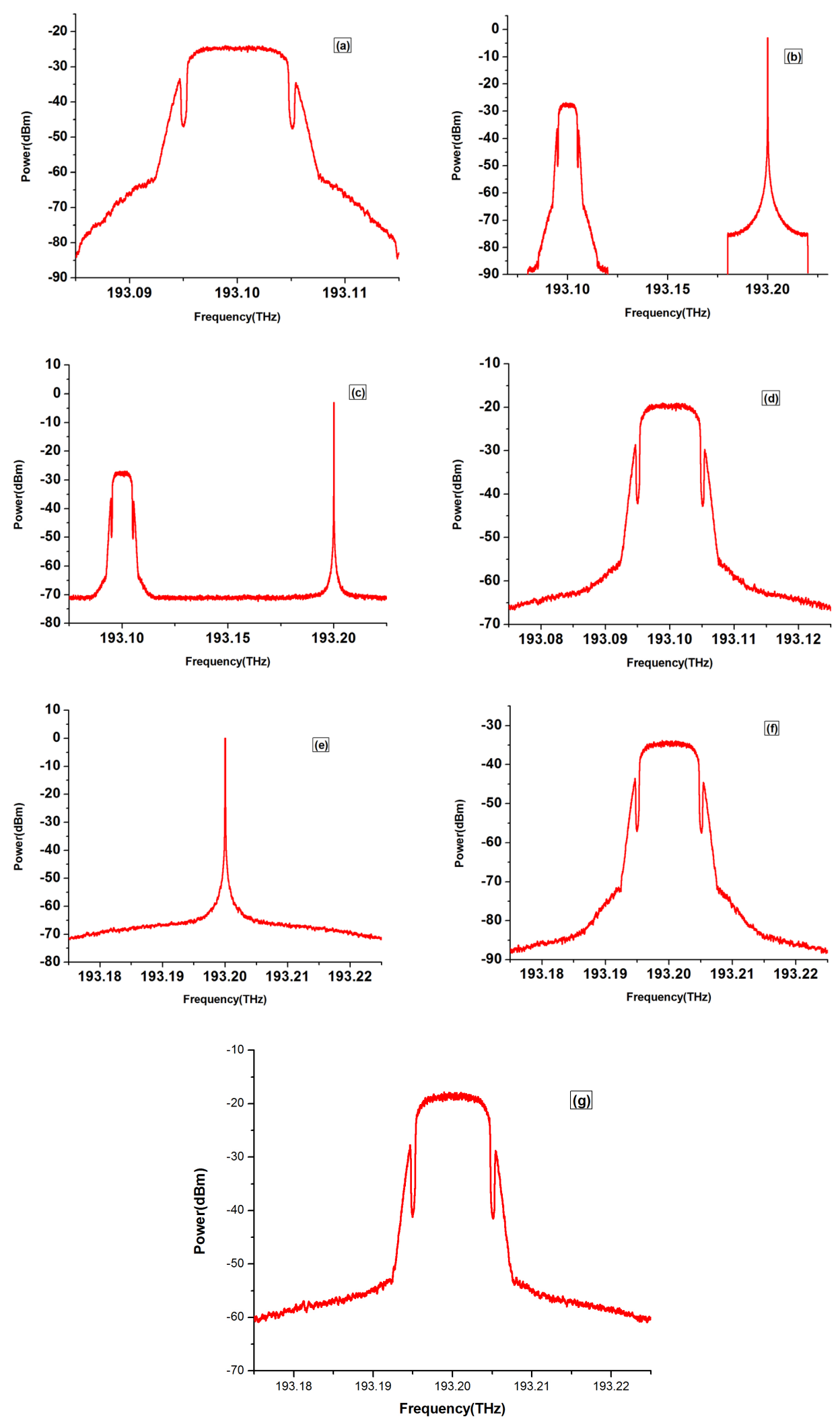

Figure 4. Optical spectra after: (a) In-phase/quadrature at the OLT; (b) OC at the OLT; (c) OS at the ODN; (d) BOF1 at the ONU; (e) BOF2 at the ONU; (f) In-phase/quadrature at the ONU; (g) BOF3 at the OLT. 
optical spectrum after 20-km SSMF transmission and OS is studied in Figure 4(c), because of EDFA, the two signals is commendably connected. Afterwards, the optical spectrum after BOF1 and BOF2 are successfully depicted in Figure 4(d) and Figure 4(e), respectively. Figure 4(f) presents the optical spectrum after in-phase/ quadrature modulation for uplink part. The optical spectrum after BOF3 is gave in Figure 4(g), which has bandwidth of $10 \mathrm{GHz}$ more than power of $20 \mathrm{dBm}$.

Figure 5 indicates the constellation diagrams after carrier phase estimation based on pilot symbols for both downlink and uplink. The constellation diagrams of 64/16/4QAM OFDM signal are experimentally measured at the received optical power of $-11.89-\mathrm{dBm},-24.11-\mathrm{dBm}$, and $-34.12-\mathrm{dBm}$, namely, log of BER are about -3.19 , -3.18 , and -3.05 , respectively, meanwhile, they are $-15.78-\mathrm{dBm},-18.97-\mathrm{dBm}$, and $-20.47-\mathrm{dBm}$ for 16/8/QPSK OFDM signal, namely, log of BER are about $-3.38,-3.22$, and -3.15 , respectively. It can be concluded from Figure 5 that MPSK is more sensitive to phase shift than MQAM, where the original transmitted data have been well recovered after channel estimation and the bit error between before and after carrier phase estimation can be basically eliminated in the modulation scheme.

As can be seen in Figure 6, the relationship between the surveying BER and the ROP for downstream and upstream in $40-\mathrm{Gb} / \mathrm{s}$ coherent detection system is experimentally measured in this pollution highlighted in Figure 6(a) and Figure 6(b), respectively. In addition, the net sequence length for hybrid 64/16/4QAM OFDM are 15360, 10240, and 5120, respectively, at the same time, they are 5120, 7680, and 10240 for hybrid QPSK/8PSK/16PSK OFDM, respectively. For a specific BER, $\log$ of BER is -3 , the receiver sensitivity of 64/16/4QAM-OFDM signal are about $-13.5,-24.5$, and -34-dBm, respectively, meanwhile, 16/8/QPSK-OFDM signal for uplink transmission are about $-16,-19.5$, and $-21-\mathrm{dBm}$, respectively.

\section{Conclusion}

A 40-Gb/s full-duplex PON infrastructure has been successfully propose and simulation demonstrate over 20km SSMF, which be significantly characterized as both hybrid modulation 64/16/4QAM OFDM signal for the downlink and hybrid modulation 16/8/Q-PSK OFDM signal for the uplink, remaining the inherent advantages introduced by orthogonal, high IFFT/FFT size, high-level QAM (MQAM) and coherent detection. Because ODN is free source, signal coming from different modulation technique can be independently transceiver for both downstream and upstream.

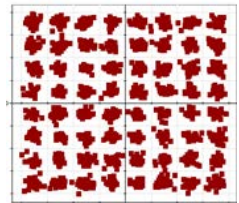

(a)

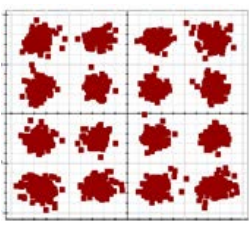

(b)

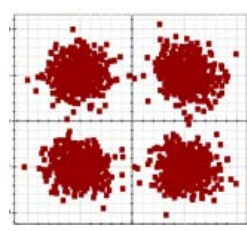

(c)

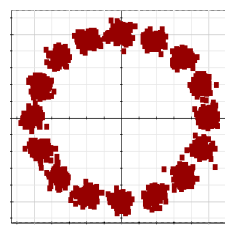

(d)

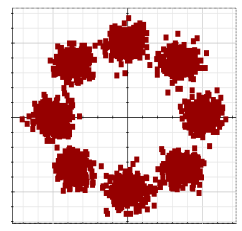

(e)

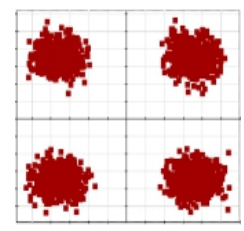

(f)

Figure 5. Constellation diagrams before after CPE for (a) 64QAM; (b) 16QAM; (c) 4QAM; (d) 16PSK; (e) 8PSK; (f) QPSK.

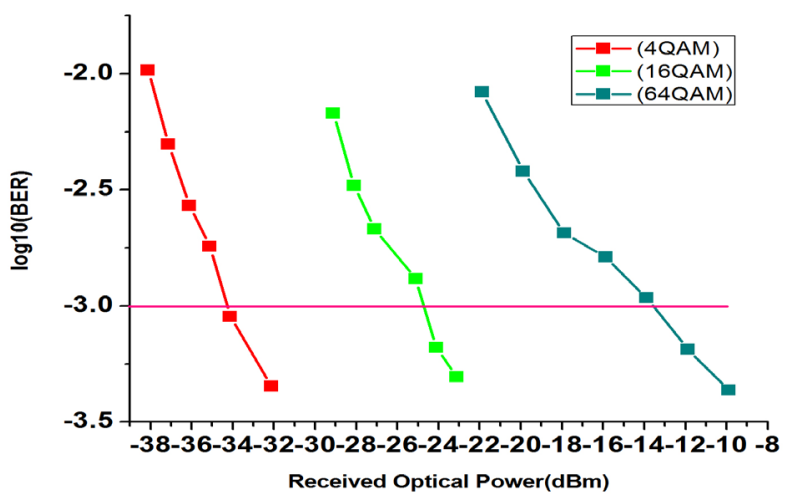

(a)

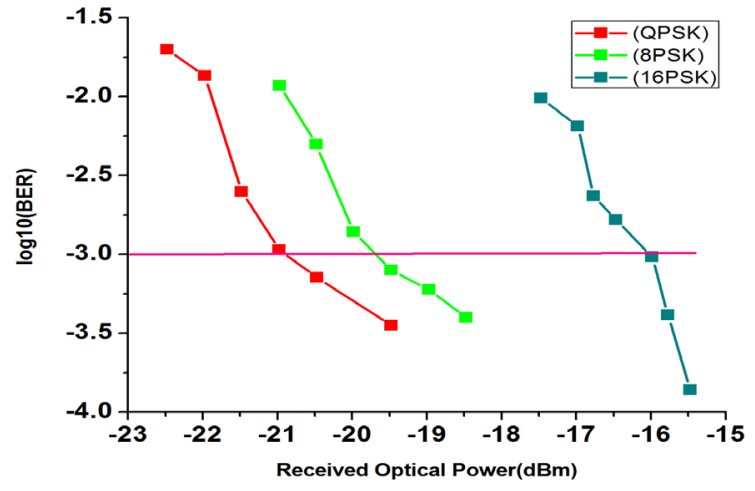

(b)

Figure 6. Measured BER of (a) downstream and (b) upstream signals versus received optical power. 


\section{Acknowledgements}

This project was supported by the National Natural Science Foundation of China (No. 61107064), the Chongqing University Innovation Team Founding (No. KJTD201320), and the Chongqing Graduate Scientific Research Innovation Project (No. CYS15225).

\section{References}

[1] Lee, C.H., Sorin, W.V. and Kim, B.Y. (2006) Fiber to the Home Using a PON Infrastructure. Journal of Lightwave Technology, 24, 4568-4583. http://dx.doi.org/10.1109/JLT.2006.885779

[2] Newaz, S.S., Cuevas, A., Lee, G.M., Crespi, N. and Choi, J.K. (2013) Improving Energy Saving in Time-Division Multiplexing Passive Optical Networks. IEEE Internet Computing, 17, 23-31. http://dx.doi.org/10.1109/MIC.2012.123

[3] Amaral, G.C., Herrera, L.E., Vitoreti, D., Temporao, G.P., Urban, P.J. and von der Weid, J.P. (2014) WDM-PON Monitoring with Tunable Photon Counting OTDR. IEEE Photonics Technology Letters, 26, 1279-1282. http://dx.doi.org/10.1109/LPT.2014.2320871

[4] Zhou, Z., Bi, M., Xiao, S., Zhang, Y. and Hu, W. (2015) Experimental Demonstration of Symmetric 100-Gb/s DML-Based TWDM-PON System. IEEE Photonics Technology Letters, 27, 470-473. http://dx.doi.org/10.1109/LPT.2014.2377271

[5] Zhou, J., Xu, F., Sun, E., Zhang, T., Zhang, Z., Guo, M., Tang, X., Wang, Z. and Qiao, Y. (2016) Coherent Optical Interleaved SC-FDM Uplink Scheme for Long-Reach Passive Optical Network. IEEE Photonics Journal, 8, 1-9.

[6] Straullu, S., Chang, J., Cigliutti, R., Ferrero, V., Nespola, A., Vinci, A., Abrate, S. and Gaudino, R. (2015) SingleWavelength Downstream FDMA-PON at 32 Gbps and 34 dB ODN Loss. IEEE Photonics Technology Letters, 27, 774-777. http://dx.doi.org/10.1109/LPT.2015.2392151

[7] Hu, T., Li, J., Ren, F., Tang, R., Yu, J., Mo, Q., Ke, Y., Du, C., Liu, Z., He, Y. and Li, Z. (2016) Demonstration of Bidirectional PON Based on Mode Division Multiplexing. IEEE Photonics Technology Letters, 28, 1201-1204. http://dx.doi.org/10.1109/LPT.2016.2530663

[8] Armstrong, J. (2009) OFDM for Optical Communications. Journal of Lightwave Technology, 27,189-204. http://dx.doi.org/10.1109/JLT.2008.2010061

[9] Cvijetic, N. (2012) OFDM for Next-Generation Optical Access Networks. Journal of Lightwave Technology, 30, 384-398. http://dx.doi.org/10.1109/JLT.2011.2166375

[10] Zhang, W., Zhang, C., Chen, C., Jin, W. and Qiu, K. (2016) Joint PAPR Reduction and Physical Layer Security Enhancement in OFDMA-PON. IEEE Photonics Technology Letters, 28, 998-1001.

[11] Cheng, M., Deng, L., Gao, X., Li, H., Tang, M., Fu, S., Shum, P. and Liu, D. (2015) Security-Enhanced OFDM-PON Using Hybrid Chaotic System. IEEE Photonics Technology Letters, 27, 326-329. http://dx.doi.org/10.1109/lpt.2014.2370757

[12] Kim, S.Y., Kani, J.I., Suzuki, K.I. and Otaka, A. (2014) OLT Receiver for Power Normalization of Burst OFDM Signals Enabling OFDM/TDMA-PON. IEEE Photonics Technology Letters, 26, 2469-2472. http://dx.doi.org/10.1109/LPT.2014.2359016

[13] Nunes, R., Shahpari, A., Silva, J., Lima, M., de Brito Andre, P. and Segatto, M. (2016) Experimental Demonstration of a 33.5-Gb/s OFDM-Based PON with Subcarrier Pre-Emphasis. IEEE Photonics Technology Letters, 28, 860-863. http://dx.doi.org/10.1109/LPT.2016.2515060 


\section{Submit or recommend next manuscript to SCIRP and we will provide best service for you:}

Accepting pre-submission inquiries through Email, Facebook, LinkedIn, Twitter, etc.

A wide selection of journals (inclusive of 9 subjects, more than 200 journals)

Providing 24-hour high-quality service

User-friendly online submission system

Fair and swift peer-review system

Efficient typesetting and proofreading procedure

Display of the result of downloads and visits, as well as the number of cited articles

Maximum dissemination of your research work

Submit your manuscript at: http://papersubmission.scirp.org/ 